\title{
Differential expression of mucins in Middle Eastern patients with colorectal cancer
}

\author{
KHAYAL AL-KHAYAL $^{1 *}$, MAHA ABDULLA $^{1 *}$, OMAR AL-OBAID $^{1}$, AHMAD ZUBAIDI $^{1}$, \\ MANSOOR-ALI VAALI-MOHAMMED ${ }^{1}$, ABDULMALIK ALSHEIKH ${ }^{2}$ and REHAN AHMAD ${ }^{1}$ \\ ${ }^{1}$ Colorectal Research Centre, Department of Surgery; ${ }^{2}$ Department of Pathology, King Khalid University Hospital, \\ College of Medicine, King Saud University, Riyadh 11472, Kingdom of Saudi Arabia
}

Received January 21, 2015; Accepted March 8, 2016

DOI: $10.3892 / 01.2016 .4672$

\begin{abstract}
Mucin overexpression has been implicated in the tumorigenesis and progression of colorectal carcinoma (CRC). However, data obtained on the prognostic importance of mucin expression in CRC is inconsistent. Due to lack of data on mucin expression and the increase in CRC incidence in Saudi Arabia, the aim of the present study was to analyze the mucin expression profile in patients with CRC in this ethnic group. The present study consisted of 22 patients that underwent surgery for CRC. Histopathological and immunohistochemical staining was performed on CRC tumor and adjacent normal tissues. A tissue microarray was prepared from the tumor and normal adjacent samples to investigate the mucin expression profile using immunohistochemistry. Formalin-fixed paraffin-embedded human colorectal cancer tissues were immunostained with mucin 1 (MUC1), mucin 2 (MUC2) and mucin 5AC (MUC5AC) antibodies. Associations between mucin expression and histopathological variables were evaluated. The present study indicated that MUC1 was highly expressed in early (stage I and II; P=0.0016) and late (stage III and IV; $\mathrm{P}<0.0001)$ stage $\mathrm{CRC}$ tissues compared to normal adjacent tissues. However, MUC2 expression was observed to be downregulated in early and late stage CRC tissues compared to normal and adjacent tissues. Furthermore, serum MUC1 levels were observed to be increased in early and late stage CRC. The present findings indicate that MUC1 expression was significantly higher in early and late stage CRC tissues and MUC2 was downregulated in CRC tissues compared with normal adjacent tissues, and serum MUC1 protein was significantly higher in $\mathrm{CRC}$ patients compared to
\end{abstract}

Correspondence to: Dr Rehan Ahmad, Colorectal Research Centre, Department of Surgery, King Khalid University Hospital, College of Medicine, King Saud University, P.O. Box 7805 (37), Riyadh 11472, Kingdom of Saudi Arabia

E-mail: arehan@ksu.edu.sa

*Contributed equally

Key words: colorectal cancer, mucin, MUC1, MUC2, MUC5AC, tissue microarray, immunohistochemistry control serum. In conclusion, during colorectal tumorigenesis the pattern of MUC1 and MUC2 expression is altered in Saudi Arabian patients with CRC compared with normal. A higher expression of MUC1 may be used as an independent biomarker in various stages of CRC tumors, which would aid in the early detection of CRC.

\section{Introduction}

The mucin family of proteins are high-molecular-weight glycoproteins, which provide protection for the epithelial cell lining that is exposed to the external environment (1). The mucin family is classified into secreted and transmembrane mucins, and is involved in the protection of the epithelial lining of the gastrointestinal and respiratory tracts and duct linings present in the pancreas, kidney, liver and mammary gland. Secreted mucins form a mucous layer that provides a physical barrier, and transmembrane mucins provide the physical barrier with ectodomains that are made up of O-glycosylated tandem repeats (1). Transmembrane mucins extend inside the cell through single membranous regions and the cytoplasmic tail of the mucins transduce signals to promote the growth and survival of the cell in response to stress (1).

Mucin 1 (MUC1) is a transmembrane glycoprotein that is overexpressed in various types of human carcinoma, including breast, colon, lung and prostate cancer (2). MUC1 is synthesized as a single polypeptide that undergoes auto-cleavage into two subunits, MUC1-N and MUC1-C, which form a stable heterodimer at the cell surface (3). The MUC1-N terminal subunit contains variable numbers of glycosylated tandem repeats (4), while the MUC1-C terminal subunit consists of a 58-amino acid extracellular domain, 28-amino acid transmembrane domain and a 72-amino acid cytoplasmic domain (2). MUC1 expression is induced by certain cytokines, including tumor necrosis factor (TNF) $\alpha$, interferon (IFN) $\gamma$ and interleukin (IL)-6. Alterations in MUC1 expression may contribute to chronic inflammation and cancer. The activation of the nuclear factor kappa-light-chain-enhancer of activated B cells $(\mathrm{NF}-\kappa \mathrm{B})$ pathway is a clear mediator of inflammation-induced cancer progression (5), and MUC1-C has been demonstrated to bind I $\mathrm{B}$ kinase (IKK) $\beta(6)$ and NF- $\mathrm{BB}$ p65 (7). Helicobacter pylori infection has been associated with MUC1 expression in inducing inflammation and the development of gastric cancer (8). In 
addition, MUC1 interacts with $\beta$-catenin and contributes to the activation of Wnt target genes leading to tumorigenesis (9). Prolonged MUC1 activation in chronic inflammation leads to growth and survival of cells undergoing a stress response (8).

The synthesis of secretory mucins is also regulated by cytokines, growth factors and bacterial products $(10,11)$. Mucin 2 (MUC2) is a secretory mucin that forms the major component of the intestinal mucus lining (1). MUC2 is primarily expressed in colorectal goblet cells. Deregulation of MUC2 expression at the epithelial cell surface provides a microenvironment where bacteria initiate an inflammatory response (12). Ulcerative colitis is a major inflammatory bowel disease (IBD), which is characterized by significant inflammation and depletion of mucin from goblet cells (13). Therefore, IBD-associated chronic inflammation increases the risk factor for colorectal cancer (CRC), potentially by promoting genomic instability in a microenvironment (14). Overexpression of MUC2 and other secreted mucins by tumors also protects tumor cells from recognition by anti-tumor immune effectors and therefore contributes to cell transformation leading to cancer (15). MUC2 in combination with mucin 5AC (MUC5AC) is clustered on chromosome 11 (16). MUC5AC is predominantly expressed in the mucus lining of the stomach and lung. Alterations in MUC2 and MUC5AC expression are reported in lung, gastrointestinal, pancreatic and liver cancer (17). MUC5AC is downregulated in non-small cell lung carcinoma (NSCLC) (18). Sialyl Lewis x antigen expression is associated with MUC5AC in NSCLC, with those patients exhibiting a poor survival time (19). MUC5AC expression in liver cancer has been shown to be associated with high lymph node metastasis (20). Alterations in mucin expression may be significantly associated with histological grade, clinical staging and prognosis of patients with CRC (17).

The majority of studies have reported that the expression of mucins in CRC is primarily confined to the late stage of the disease $(20,21)$. The incidence of CRC is increasing in young patients, specifically in the Middle East $(22,23)$. The average age of CRC detection in Saudi Arabia was 58 years between the years 2000-2006; this is younger than the age of CRC detection in the UK between the years 1996-2004, which was 68 years (22). Demographically, this indicates that the occurrence of CRC develops earlier in patients from Saudi Arabia. Although no studies have currently reported on CRC detection in young patients, the publication of the present study, and others, may mean that similar studies become more frequent. Consequently, a biomarker is required to identify CRC in young patients from Saudi Arabia. Due to the lack of data on mucin expression in Saudi CRC patients, the present study aimed to analyze the mucin expression profile in this ethnic group. The study presented the expression profile of MUC1, MUC2 and MUC5AC in various stages of CRC tissue using immunohistochemical staining. To the best of our knowledge, this is the first study where transmembrane MUC1 and secretory MUC2 and MUC5AC expression has been compared at various stages of CRC in patients from Saudi Arabia.

\section{Materials and methods}

Patient samples. The present study consisted of 22 patients that underwent surgical resection of histologically confirmed
CRC at King Khalid University Hospital, King Saud University (Riyadh, Saudi Arabia) between November 2012 and November 2013. The demographics of the patients, including age, gender, tumor site and histological stages were recorded in a database according to the Union International Contre le Cancer-Tumor-Node-Metastais Staging System and grading of CRC was in accordance with WHO classification (24-26). Histologically adjacent normal tissue from the margins of the tumors served as control tissue. All tissue samples were diagnosed and classified by two pathologists and one expert pathologist from the Department of Pathology, King Khalid University Hospital, King Saud University. In order to minimize the effect of radiotherapy and chemotherapy, all patients that had undergone neoadjuvant or adjuvant therapy were excluded from the present study. Control breast tumor samples and blood samples were obtained from the Department of Surgery, King Khaled University Hospital, King Saud University. Control blood samples were obtained from Colorectal Research Center, Department of Surgery, College of Medicine, King Khaled University Hospital, King Saud University.

The present study was approved by the Ethics Committee of King Saud University. Written informed consent was obtained from the patients for this study.

Tissue microarray (TMA). The TMA was performed as previously described (27). Formalin-fixed paraffin-embedded (FFPE) CRC tissue blocks of the 22 patients were retrieved from the archives of King Khalid University Hospital. The TMA was performed on the FFPE tumor blocks using a manual tissue arrayer (Arraymold Tissue Microarrayer kit D; IHC World LLC, Woodstock, MD, USA). Invasive carcinoma areas were identified using hematoxylin (Leica Biosystems, Inc., Buffalo Grove, IL, USA) and eosin Y (Sigma-Aldrich, St. Louis, MO, USA) (H\&E) stained slides by the aforementioned expert pathologist from the Department of Pathology, King Khalid University Hospital, King Saud University. To construct the TMA a $1 \mathrm{~mm}$ diameter needle was used to take three cores from each FFPE block corresponding to the rich tumor areas detected on the H\&E slide. The cores were inserted into the TMA paraffin block, which were subsequently incubated at $37^{\circ} \mathrm{C}$ for $30 \mathrm{~min}$ to enhance the adhesion between the cores and paraffin. TMA blocks were micro-dissected into $5-\mu \mathrm{m}$ thick sections using a semi-automatic microtome (Leica RM223; Leica Microsystems GmbH, Wetzlar, Germany), and mounted on glass slides.

Immunohistochemistry. Immunohistochemistry was performed as previously described (28). The slides with $5-\mu \mathrm{m}$ sections of tumor tissues and adjacent normal tissues were deparaffinized in xylene (Sigma-Aldrich) and rehydrated using a graded ethanol series (Sigma-Aldrich). Antigen was retrieved by boiling the slides in a microwave oven for $15 \mathrm{~min}$ in $0.01 \mathrm{~mol} / \mathrm{l}$ citrate buffer ( $\mathrm{pH}$ 6.0; Sigma-Aldrich). Endogenous peroxidase was blocked with a $3 \% \mathrm{H}_{2} \mathrm{O}_{2}$-methanol solution (Sigma-Aldrich), and the slides were incubated in $10 \%$ normal goat serum (Sigma-Aldrich) for $30 \mathrm{~min}$ to prevent nonspecific staining. The tissue sections were incubated overnight at $4^{\circ} \mathrm{C}$ with the following primary antibodies from Santa Cruz Biotechnology, Inc. (Dallas, TX, USA) at a dilution of 1:100: Rabbit polyclonal anti-MUC1 (catalog no., sc-15333), rabbit 
polyclonal anti-MUC2 (catalog no., sc-15334) or mouse monoclonal anti-MUC5AC (catalog no., sc-33667). The standard biotin-streptavidin-peroxidase method was subsequently used with affinity purified goat anti-mouse IgM secondary antibody (catalog no., RE7103; dilution, $5 \mu \mathrm{g} / \mathrm{ml}$; Leica Biosystems, Inc.), and the sections were lightly counterstained with hematoxylin. Breast tumor was histologically used as positive controls for MUC1, MUC2 and MUC5AC. As a negative control, the same procedure was conducted without primary antibody. The expression of MUC1, MUC2 and MUC5AC in tumor and adjacent normal samples was analyzed using an eSlide capture device (Aperio CS2; Leica Microsystems GmbH).

Image analysis. High-resolution, whole-slide digital scans of all TMA glass slides were created using the eSlide capture device. The digital slide images were viewed by ScanScope slide scanner (ScanScope CS; Aperio ImageScope; Leica Microsystems $\mathrm{GmbH}$ ), and analyzed using Aperio's Image Analysis Algorithms (Leica Microsystems GmbH). For each core, five fields of $0.2645 \mu \mathrm{m}^{2}$ were randomly selected. Color Deconvolution Algorithm (Aperio; Leica Microsystems $\mathrm{GmbH}$ ) was run on the selected area and this generated an intensity range color markup image, segmenting and color-coding various parts of the image according to the intensity of positive staining. The area for each of these four intensity categories (expressed as a percent relative to the total analysis area), together with the average positive intensity and the average optical density, was also provided as numerical output. The algorithm output also included a score (0-60) of mucin expression based on the percent strong positive and percent medium positive. These values were combined and named as 'percent strong positive'. The analysis output results were exported to Excel 2010 spreadsheets (Microsoft Corporation, Redmond, WA, USA) and subjected to statistical analysis, focusing primarily on the percentage of the total positive cells as the parameters to be statistically analyzed and compared.

Serum MUC1 determination. Serum MUC1 levels were measured using Human Carbohydrate Antigen 15-3 / Mucin-1 ELISA kit (catalog no., RAB0375; Sigma-Aldrich), according to the manufacturer's protocol.

Statistical analysis. Data was presented with mean \pm standard deviation. Statistical analysis was performed using Microsoft Excel (Microsoft Corporation). The means between the CRC tissue and normal adjacent tissue were compared using Student's t-test. $\mathrm{P} \leq 0.05$ was considered to indicate a statistically significant difference.

\section{Results}

Clinicopathological features of the patients. In total, $\sim 90 \%$ of the tumor samples (20/22) were advanced CRC at stage II and III. Additionally, the majority of the tumors were high grade (grade 2 and Grade 3). A total of 8 patients out of 22 already had developed lymph node metastases (Table I).

Mucin staining. Mucin protein expression in the CRC and normal adjacent tissues was observed using
Table I. Clinicopathological characteristics of patients with colorectal cancer.

\begin{tabular}{|c|c|}
\hline Characteristic & Value \\
\hline Total, n & 22 \\
\hline \multicolumn{2}{|l|}{ Gender, n (\%) } \\
\hline Male & $11(50)$ \\
\hline Female & $11(50)$ \\
\hline \multicolumn{2}{|l|}{ Age, years } \\
\hline Median & 57 \\
\hline Range & $36-81$ \\
\hline \multicolumn{2}{|l|}{ Site of cancer, n (\%) } \\
\hline Colon & $9(41)$ \\
\hline Rectum & $2(9)$ \\
\hline Sigmoid & $5(23)$ \\
\hline Rectosigmoid & $6(27)$ \\
\hline Adenocarcinoma, n (\%) & $22(100)$ \\
\hline \multicolumn{2}{|l|}{ Histological grade, n (\%) } \\
\hline 2 & $19(86)$ \\
\hline 3 & $3(14)$ \\
\hline \multicolumn{2}{|l|}{ Clinical staging, n (\%) } \\
\hline I & $1(5)$ \\
\hline II & $12(55)$ \\
\hline III & $8(36)$ \\
\hline IV & $1(5)$ \\
\hline \multicolumn{2}{|l|}{ Tumor staging, n (\%) } \\
\hline $\mathrm{T} 2$ & $3(14)$ \\
\hline $\mathrm{T} 3$ & $17(77)$ \\
\hline $\mathrm{T} 4$ & $2(9)$ \\
\hline \multicolumn{2}{|l|}{ Lymph node status, n (\%) } \\
\hline No & $14(64)$ \\
\hline $\mathrm{N} 1$ & $6(27)$ \\
\hline $\mathrm{N} 2$ & $2(9)$ \\
\hline \multicolumn{2}{|l|}{ Metastasis, n (\%) } \\
\hline Yes & $9(41)$ \\
\hline No & $13(59)$ \\
\hline
\end{tabular}

immunohistochemical staining, and the results are summarized in Table II. The expression of MUC1 and MUC2 varied between normal tissues and different stages of colorectal tumor. The majority of the staining was prominently characterized by diffuse cytoplasmic staining. MUC1 protein was observed to be weakly expressed in the adjacent normal tissue group (Fig. 1A and B). However in the colorectal tumor group, the majority of staining for MUC1 was observed within the cytoplasm and cell membrane (Fig. 1C and D). For MUC1, positive staining was observed in $82 \%(18 / 22)$ of tumor tissues and no staining was observed in $18 \%$ (4/22) tumor tissues (Table II). There was a significant difference between MUC1 positive staining in tumor and negative adjacent normal tissue $(\mathrm{P}<0.0001)$. There was clear MUC2 staining in the lumen and cytoplasm of goblet cells in adjacent normal tissue (Fig. 2A and B); however it was negative or weak in 
Table II. Frequency of mucin expression in colorectal tumors and adjacent normal tissues from 22 patients with colorectal cancer.

\begin{tabular}{lcc}
\hline Staining score & Tumor, n (\%) & Adjacent normal, n (\%) \\
\hline MUC1 & & \\
Positive & $18(82)$ & $5(23)$ \\
Negative & $4(18)$ & $17(77)$ \\
MUC2 & & $19(86)$ \\
Positive & $2(9)$ & $3(14)$ \\
Negative & $20(91)$ & 0.0005 \\
\hline
\end{tabular}

Student's t-test used for analysis of difference in expression. MUC, mucin.

Table III. Tumor stage and MUC1 expression in patients with colorectal carcinoma according to age.

\begin{tabular}{lcr}
\hline Pathological stage & Young patients, $\leq 50$ years, $\mathrm{n}(\%)$ & Old patients, >50 years, $\mathrm{n}(\%)$ \\
\hline Total & $7(31)$ & $15(68)$ \\
Clinical staging & & $10(67)$ \\
Early (I and II) & $3(43)$ & $5(33)$ \\
Late (III and IV) & $4(57)$ & $14(93)$ \\
MUC1 positive expression & $7(100)$ & \\
\hline
\end{tabular}

MUC, mucin.
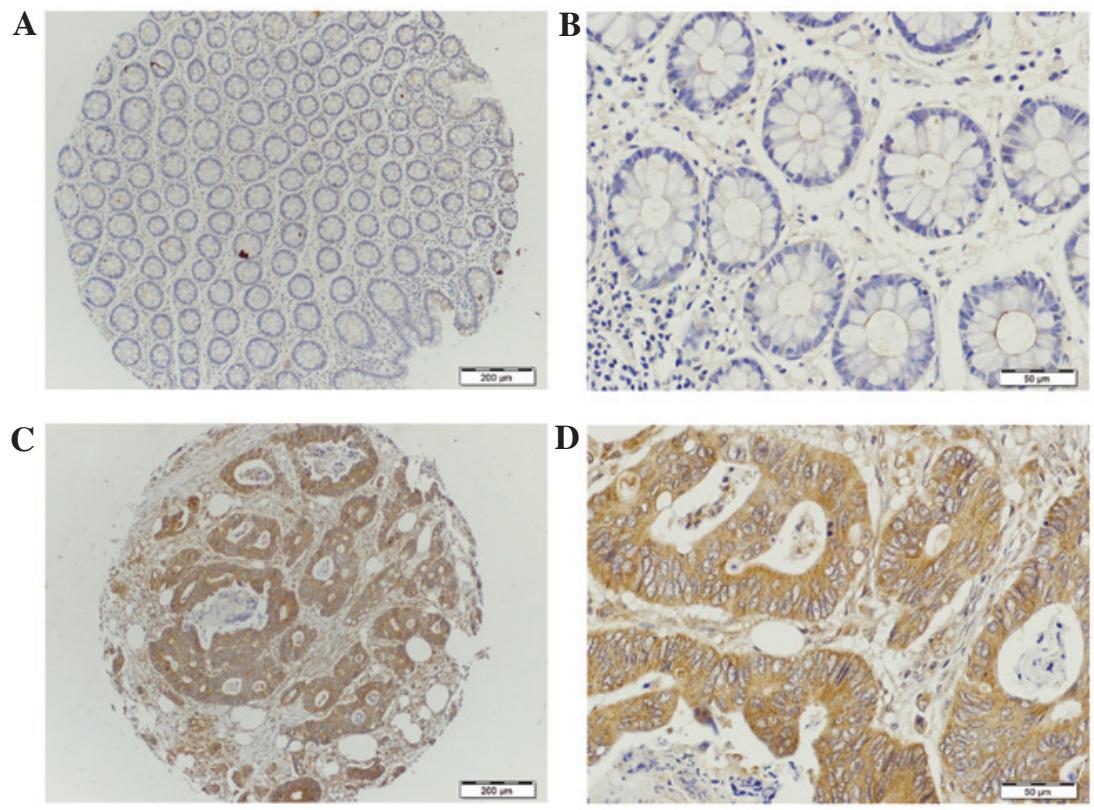

Figure 1. Immunohistochemical staining of representative tissue microarray cores with MUC1 antibody. (A and B) Negative MUC1 staining in normal adjacent tissue at x10 and x40 magnification, respectively. (C and D) Positive MUC1 staining in colorectal carcinoma tumor tissue at x10 and x40 magnification, respectively. MUC, mucin.

colorectal tumor tissues (Fig. 2C and D). The majority of the tumor tissues (90\%) exhibited negative MUC2 staining. In total, $86 \%$ of the adjacent normal tissues exhibited clear positive staining for MUC2. There was a significant difference between MUC2 negative staining in tumor tissues and positive staining in normal tissue $(\mathrm{P}=0.0005)$. The MUC5AC staining was observed to be negative in tumor and adjacent normal tissues. Collectively, these findings demonstrate that MUC1 was highly expressed in colorectal tumor compared to normal tissue, and MUC2 protein expression was downregulated in tumor tissues compared with adjacent normal tissues. Colorectal tumors did not express MUC5AC. 

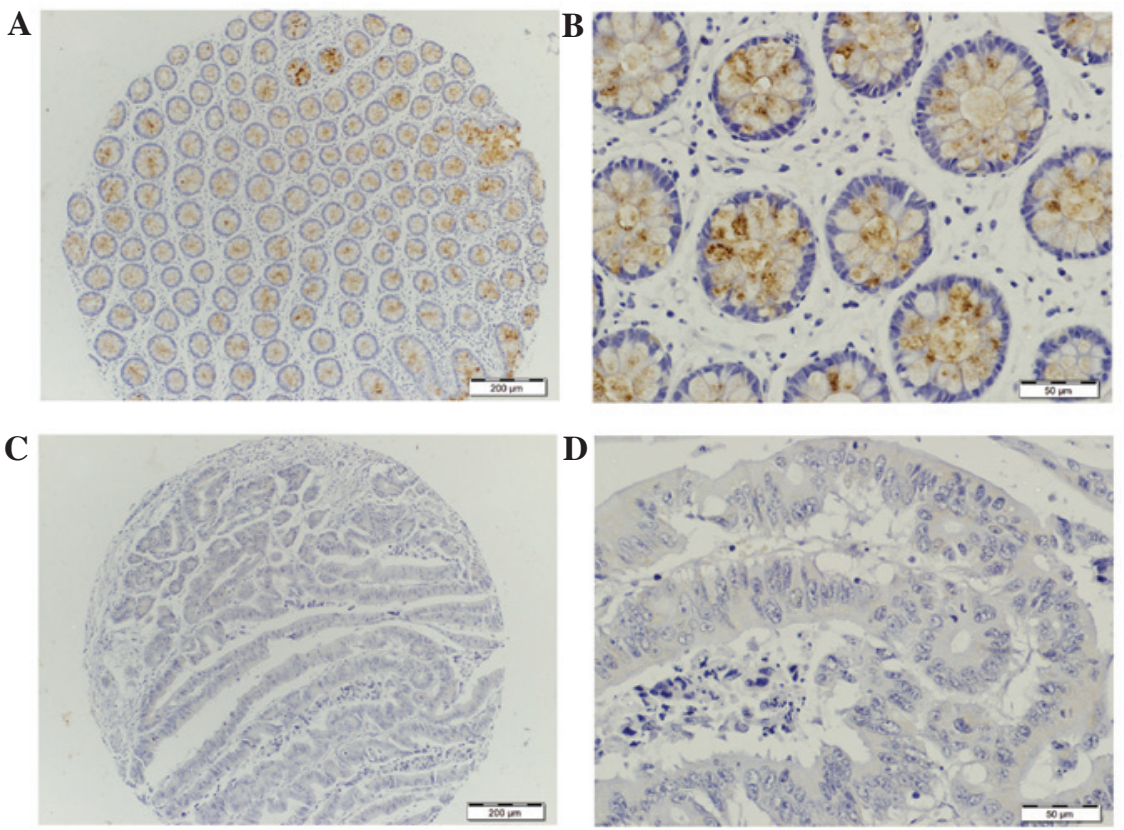

Figure 2. Immunohistochemical staining of representative tissue microarray cores with MUC2 antibody. (A and B) Positive MUC2 staining in normal adjacent tissue at x10 and x40 magnification, respectively. (C and D) Negative MUC2 staining in colorectal carcinoma tumor tissue at x10 and x40 magnification, respectively. MUC, mucin.

A

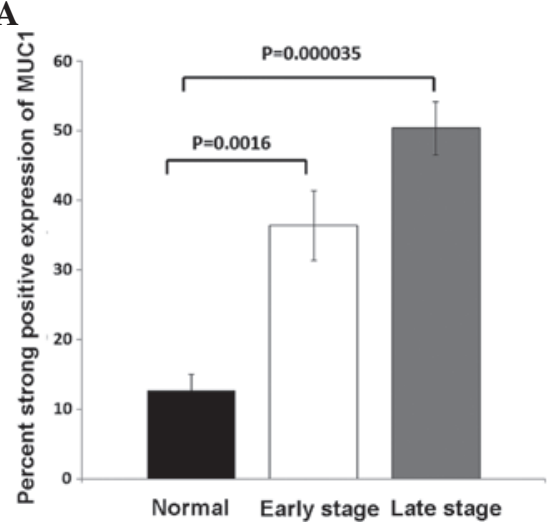

B

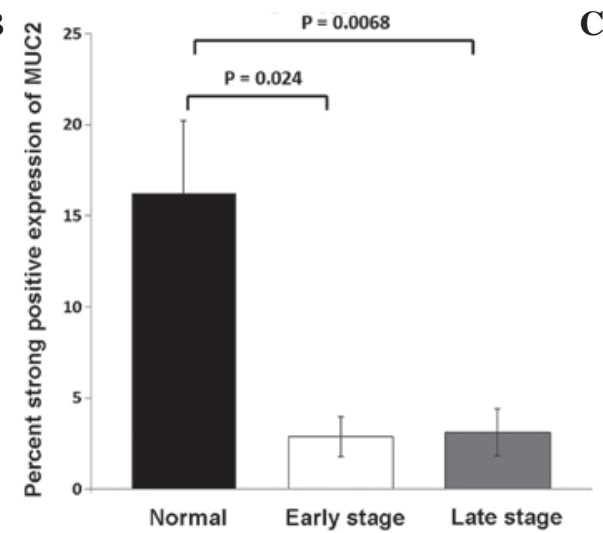

C

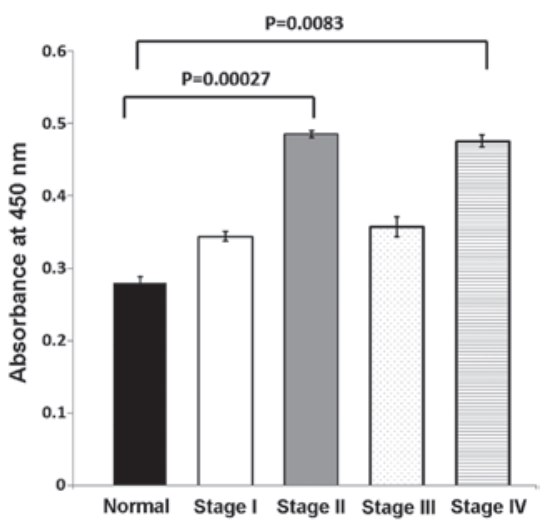

Figure 3. (A) Expression of MUC1 in normal adjacent and early and late CRC tumor tissue. (B) Expression of MUC2 in normal adjacent and early and late CRC tumor tissue. (C) Serum MUC1 levels determined by enzyme-linked immunosorbent assay in normal adjacent (black) and stage I (white), II (gray), III (dots) and IV (horizontal lines) CRC tumor tissues. MUC, mucin; CRC, colorectal carcinoma.

MUC1 positive staining was similar across all ages of CRC patients (Table III). It was observed that MUC1 expression was also positive in young CRC patients and old CRC patients from Saudi Arabia. There was no significant difference between MUC1 staining the ages of CRC patients $(\mathrm{P}=0.0820)$.

Immunohistochemical analysis of MUC1, MUC2 and $M U C 5 A C$. MUC1 was demonstrated to have an increased expression in colorectal tumor compared with adjacent normal tissue. In adjacent normal tissue, MUC1 staining was extremely weak (Fig. 1A and B). MUC1 expression in colorectal tumor was clearly observed to be localized in the cytoplasm (Fig. 1C and D). The expression frequency and strong positive staining of MUC1 was significantly higher in early (stage I and II) and late stage (stage III and IV) tumor tissue compared with adjacent normal tissue (Fig. 3A). When
MUC1 expression was compared between adjacent normal and early stage tumor tissue, MUC1 was found to be overexpressed $(\mathrm{P}=0.0016)$. However, MUC1 expression was significantly increased in late stage colorectal tumors compared with normal adjacent tissue $(\mathrm{P}<0.0001)$. The frequency of MUC2 strong positive staining was increased in adjacent normal tissue compared with colorectal tumor tissue. MUC2 staining was primarily in the lumen and cytoplasm of adjacent normal tissue (Fig. 2A and B). MUC2 positive staining was found to be significantly downregulated in early stage $(\mathrm{P}=0.0240)$ and late stage $(\mathrm{P}=0.0068)$ colorectal cancer compared with adjacent normal tissue (Fig. 3B). The MUC2 expression profile for colorectal tumor and adjacent normal tissue identified in the present study are consistent with other reports $(17,29)$. In the present study, MUC5AC expression was undetected in tumor and adjacent normal tissue (data not shown). These 
results demonstrate that MUC1 was highly expressed in tumor samples and localized in the cytoplasm; however, MUC2 expression was high in normal samples and was decreased in tumor samples. Therefore, MUC1 and MUC2 expression are inversely associated in Saudi patients with CRC and these colorectal tumors are negative for MUC5AC expression.

Serum MUC1 levels in colorectal tumor. Serum MUC1 levels were measured in control individuals and patients with various stages of CRC (stage I, II, III and IV). MUC1 serum levels were increased in stage II $(\mathrm{P}=0.0003)$ and stage IV $(\mathrm{P}=0.0083)$ serum compared with control serum (Fig. 3C). Serum MUC1 levels were associated with higher MUC1 staining in early and late stage CRC. These findings indicate that MUC1 is highly expressed in early and late stage tumors and was demonstrated to be cleaved during early and late stage CRC. Overall, the present results collectively indicated that MUC1 is clearly expressed in CRC tissues and MUC2 is downregulated during tumor transformation in CRC. MUC1 gene expression was demonstrated to be increased in tumor tissue and serum MUC1 levels were high in early and late stage patients with CRC.

\section{Discussion}

Mucins are high molecular weight glycoproteins with 20 amino acid tandem repeats, which undergo glycosylation (1). Mucins are known to be aberrantly expressed in CRC with aggressive phenotypes $(17,20,21)$. However, the mucin expression profile of patients with CRC is not known in the Middle East, specifically in Saudi Arabia and, to the best of our knowledge, there has only been one report that studied the impact of mucin production on the prognosis of patients with CRC (30). Therefore, the present study investigated the expression of MUC1, MUC2 and MUC5AC as a biomarker in various stages of CRC in Saudi patients. In the present study population, $\sim 40 \%$ of tumors were identified in the colon, and $\sim 60 \%$ were in the rectum, sigmoid and rectosigmoid. All these tumors were high-grade adenocarcinomas consisting of early stage (stage I and II, 60\%) and late stage (stage III and IV, $\sim 40 \%$ ). In total, $\sim 37 \%$ of the patients were positive for lymph node metastases. Almost $81 \%$ of the CRC tumor samples were positive for MUC1 in the present study. The majority of these positive MUC1 tumors expressed MUC1 in the cytoplasm of tumor cells. Adjacent normal tissues from the same patients were negative for MUC1 staining. In addition, MUC1 staining was positive in patients with early-stage CRC (stage I and II; $\mathrm{P}=0.0016$ ) compared with normal adjacent tissues. This is in contrast to previous studies performed in Europe, which reported that MUC1 is only upregulated in late-stage metastatic CRC (20,31); however, in the present study, it is significantly higher in late stage tumor $(\mathrm{P}<0.0001)$. In the present study, the expression of $\mathrm{MUC1}$ in late stages is significantly higher than early stage colorectal cancer $(\mathrm{P}=0.0460)$. It appears that the expression profile of MUC1 in Saudi CRC patients is different, since there is MUC1 expression in early-stage CRC tumors. Therefore, MUC1 expression may be dependent on ethnicity and demographic location of individuals.

MUC1 forms the mucosal barrier of the intestinal tract and protects the epithelial lining from ingested toxins, pathogenic bacteria and inflammatory cytokines (1). However, pro-inflammatory cytokines, including TNF $\alpha$, IFN $\gamma$ and IL-6, are known to induce MUC1 expression during chronic inflammation. Overexpression of MUC1 during chronic inflammation induces pro-tumorigenic effects and leads to the development of CRC $(1,15)$. The IKK $\beta-N F \kappa B$ pathway is a primary mediator of inflammation-induced cancer progression (1), and notably, MUC1 binds IKK $\beta$ (6) and NFKB p65 (7) and contributes to the constitutive activation of the $\mathrm{NF} \kappa \mathrm{B}$ pathway. In this regard, during chronic inflammation MUC1-induced activation of the $\mathrm{NF} \times \mathrm{B}$ pathway may lead to CRC. In addition, MUC1 interacts with $\beta$-catenin during cell adhesion (9). NFKB is constitutively activated in $\mathrm{CRC}$, which is important in promoting tumor growth (32), and $\beta$-catenin is has been demonstrated to be involved in CRC tumorigenesis (33). Consequently, it is possible that MUC1 may be involved in activating $\beta$-catenin and $\mathrm{NF \kappa B}$ signaling pathways; thereby contributing to $\mathrm{CRC}$ progression.

The expression of the secretory mucin MUC2 is restricted to normal tissue, mostly in the lumen and cytoplasm. In the present study, MUC2 was expressed in $>85 \%$ of adjacent normal tissue, and was not expressed or had an extremely low expression in early and late stage CRC tumor tissues when compared with normal tissue $(\mathrm{P}=0.0240$ and $\mathrm{P}=0.0068$, respectively). This is consistent with other studies that demonstrated that there was a reduced expression of MUC2 in patients with CRC $(17,29,31,34)$. This decrease or absence of MUC2 expression may be due to MUC2 promoter methylation in CRC cells (35). A reduced MUC2 expression in colon cancer contributes to the pro-survival pathway in cells; 553 protein regulates MUC2 transcription as MUC2 staining is reported to be inversely associated with p53 expression in mucinous carcinoma (36). The intestinal epithelial lining is covered by a mucous layer, which is primarily composed of secreted mucins. This mucous layer acts as a barrier that prevents the epithelial lining from damage and blocks the activation of the immune response. MUC2 is a primary component of the mucous layer of the normal intestine. In the absence of MUC2, an inflammatory process is initiated at the cell surface, and a loss of MUC2 in goblet cells during chronic inflammation is associated with ulcerative colitis (1). Therefore, depletion of MUC2 production results in the increased risk of colorectal cancer.

In the present study, MUC5AC was not identified in either normal adjacent or CRC tumor tissues. This is not notable considering that previous studies have demonstrated that MUC5AC is not expressed in poorly-differentiated colorectal tumors $(16,17,19,21)$. By contrast, MUC5AC has been reported to be expressed in well-differentiated tumors $(17,21)$. CRC patients with MUC5AC negative tumors have a poor prognosis with a low survival rate compared with patients with MUC5AC positive tumors $(17,21)$. This indicates that an absence of MUC5AC expression is a prognostic factor for highly aggressive colorectal cancer.

The majority of the present study sample consisted of patients with higher grade colorectal tumors. Mucin expression has been correlated with high levels of microsatellite instability, in particular an increased expression of MUC2 and MUC5AC in sporadic cancer (31). Mucin gene expression may contribute to cell transformation, and consequently 
tumorigenesis, due to the loss of tumor suppressor genes; mucin expression has been associated with mutations in mismatch repair genes or mutL homolog 1 (MLH1) hypermethylation (29). MUC1 is a well-known interacting protein that may interact with certain histone methyl transferases, and therefore lead to hypermethylation of MLH1. In the present study, serum MUC1 levels were higher in early and late stage CRC tissues compared with normal adjacent tissues. MUC1 expression has been reported in the serum of patients with head and neck squamous cell carcinoma (HNSCC); MUC1 serum levels were higher in HNSCC patients compared with control individuals, and was positively associated with MUC1 staining in the HNSCC tumors (37).

The incidence of CRC and mortality rates are decreasing among in patients aged $>50$ years worldwide; however, the incidence of CRC is increasing in young patients and is termed as young onset CRC. A similar trend has been observed in Saudi Arabia, where there has been an increase in the incidence of young onset CRC (21). Young onset CRC is characterized by microsatellite stability primarily identified in the distal colon and rectum with poor differentiation (38). Furthermore, patients with young onset CRC present with advance staged $\mathrm{CRC}$ with mucinous and signet ring features.

The present study demonstrates a role for MUC1 in the progression of CRC using a TMA in CRC patients from Saudi Arabia. Therefore, MUC1 expression may be used as an independent prognostic marker in CRC. The present findings implicate MUC1 expression as possessing diagnostic, prognostic and therapeutic significance in CRC. MUC1 overexpression in early and late stage CRC may be a useful biomarker, particularly in young onset CRC patients. Specifically in Saudi CRC patients, MUC1 appears to be expressed significantly in early stage cancer, which will aid in the early diagnosis of CRC. MUC1 expression in young CRC patients from Saudi Arabia may be due to a differences observed in the ethnic population, including genetic predisposition, diet and smoking. In conclusion, the present study demonstrates that MUC1 may be a useful biomarker for the detection of early as well as late stage $\mathrm{CRC}$. Additional studies are required with a larger sample size to evaluate the biomarker capability of MUC1 in CRC, particularly in patients with a young onset CRC phenotype.

\section{Acknowledgements}

The authors would like to thank Dr Amer Mahmood of Stem Cell Unit (King Saud University) for valuable scientific input that greatly improved this manuscript. The present study was supported by the Vice Deanship Research Chair, King Saud University Deanship of Scientific Research.

\section{References}

1. Kufe DW: Mucins in cancer: Function, prognosis and therapy Nat Rev Cancer 9: 874-885, 2009.

2. Kufe DW: MUC1-C oncoprotein as a target in breast cancer: Activation of signaling pathway and therapeutic approaches. Oncogene 32: 1073-1081, 2013.

3. Macao B, Johansson DG, Hansson GC and Härd T: Autoproteolysis coupled to protein folding in the SEA domain of the membrane-bound MUC1 mucin. Nat Struct Mol Biol 13: 71-76, 2006.
4. Siddiqui J, Abe M, Hayes D, Shani E, Yunis E and Kufe D: Isolation and sequencing of a cDNA coding for the human DF3 breast carcinoma-associated antigen. Proc Natl Acad Sci USA 85: 2320-2323, 1988.

5. Karin M and Greten FR: NF-kappaB: Linking inflammation and immunity to cancer development and progression. Nat Rev Immunol 10: 749-759, 2005.

6. Ahmad R, Raina D, Trivedi V, Ren J, Rajabi H, Kharbanda S and Kufe D: MUC1 oncoprotein activates the I kappaB kinase complex and constitutive NF-kappaB signaling. Nat Cell Biol 9: 1419-1427, 2007.

7. Ahmad R, Raina D, Joshi MD, Kawano T, Ren J, Kharbanda S and Kufe D: MUC1-C oncoprotein functions as a direct activator of the nuclear factor-kappaB p65 transcription factor. Cancer Res 69: 7013-7021, 2009.

8. Vinall LE, King M, Novelli M, Green CA, Daniels G, Hilkens J, Sarner M and Swallow DM: Altered expression and allelic association of the hypervariable membrane mucin MUC1 in Helicobacter pylori gastritis. Gastroenterology 123: 41-49, 2002.

9. Huang L, Chen D, Liu D, Yin L, Kharbanda S and Kufe D: MUC1 oncoprotein blocks glycogen synthase kinase 3 beta-mediated phosphorylation and degradation of beta-catenin. Cancer Res 65: 10413-10422, 2005.

10. Kanoh A, Takeuchi H, Kato K, Waki M, Usami K and Irimura T: Interleukin-4 induces specific pp-GalNAc-T expression and alterations in mucin $\mathrm{O}$-glycosylation in colonic epithelial cells. Biochim Biophys Acta 1780: 577-584, 2008.

11. Byrd JC and Bresalier RS: Mucins and mucin binding proteins in colorectal cancer. Cancer Metastasis Rev 23: 77-99, 2004.

12. Johansson ME, Phillipson M, Petersson J, Velcich A, Holm L and Hansson GC: The inner of the two Muc2 mucin-dependent mucus layers in colon is devoid of bacteria. Proc Natl Acad Sci USA 105: 15064-15069, 2008.

13. Xavier RJ and Podolsky DK: Unravelling the pathogenesis of inflammatory bowel disease. Nature 448: 427-434, 2007.

14. Feagins LA, Souza RF and Spechler SJ: Carcinogenesis in IBD: Potential targets for the prevention of colorectal cancer. Nat Rev Gastroenterol Hepatol 6: 297-305, 2009.

15. Hollingsworth MA and Swanson BJ: Mucins in cancer: Protection and control of the cell surface. Nat Rev Cancer 4: 45-60, 2004.

16. Vincent A, Perrais M, Desseyn JL, Aubert JP, Pigny P and Van Seuningen I: Epigenetic regulation (DNA methylation, histone modifications) of the $11 \mathrm{p} 15$ mucin genes (MUC2, MUC5AC, MUC5B, MUC6) in epithelial cancer cells. Oncogene 26: 6566-6576, 2007.

17. Bu XD, Li N, Tian XQ, Li L, Wang JS, Yu XJ and Huang PL: Altered expression of MUC2 and MUC5AC in progression of colorectal carcinoma. World J Gastroenterol 16: 4089-4094, 2010.

18. López-Ferrer A, Curull V, Barranco C, Garrido M, Lloreta J, Real FX and de Bolós C: Mucins as differentiation markers in bronchial epithelium. Squamous cell carcinoma and adenocarcinoma display similar expression patterns. Am J Respir Cell Mol Biol 24: 22-29, 2001.

19. Yu CJ, Shih JY, Lee YC, Shun CT, Yuan A and Yang PC: Sialyl Lewis antigens: Association with MUC5AC protein and correlation with post-operative recurrence of non-small cell lung cancer. Lung Cancer 47: 59-67, 2005.

20. Duncan TJ, Watson NF, Al-Attari AH, Scholefield JH and Durrant LG: The role of MUC1 and MUC3 in the biology and prognosis of colorectal cancer. World J Sur Oncol 5: 31, 2007.

21. Kocer B, Soran A, Erdogan S, Karabeyoglu M, Yildirim O, Eroglu A, Bozkurt B and Cengiz O: Expression of MUC5AC in colorectal carcinoma and relationship with prognosis. Pathol Int 52: 470-477, 2002.

22. Mosli MH and Al-Ahwal MS: Colorectal cancer in the kingdom of Saudi Arabia: Need for screening. Asian Pac J Cancer Prev 13: 3809-3813, 2012

23. Gado A, Ebeid B, Abdelmohsen A and Axon A: Colorectal cancer in Egypt is commoner in young people: Is this cause for alarm. Alexandria J Med 50: 197-201, 2014.

24. Greene FL: TNM staging for malignancies of the digestive tract: 2003 changes and beyond. Semin Surg Oncol 21: 23-29, 2003.

25. Sobin LH and Wittekind C (eds): TNM Classification of Malignant Tumours. 6th edition. John Wiley \& Sons, Hoboken, NJ, 2002.

26. Hamilton SR and Aaltonen L: Tumours of small intestine. In: World Health Organization Classification of Tumours. Pathology and Genetics of Tumours of the Digestive System. IARC Press, Lyon, pp69-91, 2000. 
27. Rimm DL, Camp RL, Charette LA, Costa J, Olsen DA and Reiss M: Tissue microarray: A new technology for amplification of tissue resources. Cancer J 7: 24-31, 2001.

28. Biemer-Hüttmann AE, Walsh MD, McGuckin MA, et al: Mucin core protein expression in colorectal cancers with high levels of microsatellite instability indicates a novel pathway of morphogenesis. Clin Cancer Res 6: 1909-1916, 2000.

29. Farhat MH, Barada KA, Tawil AN, Itani DM, Hatoum HA and Shamseddine AI: Effect of mucin production on survival in colorectal cancer: A case-control study. World J Gastroenterol 14 6981-6985, 2008.

30. Baldus SE, Mönig SP, Hanisch FG, Zirbes TK, Flucke U, Oelert S, Zilkens G, Madejczik B, Thiele J, Schneider PM, et al: Comparative evaluation of the prognostic value of MUC1, MUC2, sialyl-Lewis(a) and sialyl-Lewis(x) antigens in colorectal adenocarcinoma. Histopathology 40: 440-449, 2002.

31. Voloshanenko O, Erdmann G, Dubash TD, Augustin I, Metzig M, Moffa G, Hundsrucker C, Kerr G, Sandmann T, Anchang B, et al: Wnt secretion is required to maintain high levels of Wnt activity in colon cancer cells. Nat Commun 4: 2610, 2013.

32. Ajioka Y, Allison LJ and Jass JR: Significance of MUC1 and MUC2 mucin expression in colorectal cancer. J Clin Pathol 49: 560-564, 1996
33. Gratchev A, Siedow A, Bumke-Vogt C, Hummel M, Foss HD, Hanski ML, Kobalz U, Mann B, Lammert H, Mansmann U, et al: Regulation of the intestinal mucin MUC2 gene expression in vivo: Evidence for the role of promoter methylation. Cancer Lett 168: 71-80, 2001

34. Ookawa K, Kudo T, Aizawa S, Saito H and Tsuchida S: Transcriptional activation of the MUC2 gene by p53. J Biol Chem 277: 48270-48275, 2002.

35. Biemer-Hüttmann AE, Walsh MD, McGuckin MA, Simms LA, Young J, Leggett BA and Jass JR: Mucin core protein expression in colorectal cancers with high levels of microsatellite instability indicates a novel pathway of morphogenesis. Clin Cancer Res 6: 1909-1916, 2000

36. Lugli A, Zlobec I, Baker K, Minoo P, Tornillo L, Terracciano L and Jass JR: Prognostic significance of mucins in colorectal cancer with different DNA mismatch-repair status. J Clin Pathol 60: 534-539, 2007.

37. Rabassa ME, Croce M, Pereyra A and Segal-Eiras A: MUC expression and anti-MUC1 serum immune response in head and neck squamous cell carcinoma (HNSCC): A multivariate analysis. BMC Cancer 6: 253, 2006.

38. Ahnen DJ, Wade SW, Jones WF, Sifri R, Mendoza Silveiras J, Greenamyer J, Guiffre S, Axilbund J, Spiegel A and You YN: The increasing incidence of young onset colorectal cancer: A call to action. Mayo Clinic Proc 89: 216-224, 2014. 